\title{
Impacts Socioéconomiques Et Sanitaires De L'exploitation Agricole De La Plaine Inondable Todé- Gbamè Au Bénin
}

\author{
Séraphin Capo Atidegla \\ Faculté des Sciences Agronomiques (FSA), 01 BP 526 Cotonou, Bénin \\ Hermine Elisée Degbo \\ Faculté des Sciences et Techniques (FAST), 01 BP 526 Cotonou, Bénin \\ Thierry Azonhe \\ Faculté des Lettres, Arts et Sciences Humaines (FLASH), 01 BP 526 \\ Cotonou, Bénin \\ Euloge Kossi Agbossou \\ Faculté des Sciences Agronomiques (FSA), 01 BP 526 Cotonou, Bénin \\ Institut National de l'Eau (IINE), 01 BP 526 Cotonou, Bénin
}

doi: 10.19044/esj.2017.v13n18p185 URL:http://dx.doi.org/10.19044/esj.2017.v13n18p185

\begin{abstract}
The present study aims to determinate the prevalence of the diseases linked to the agricultural exploitation of Todé-Gbamè plain liable to flooding in Benin and to assess the related work wasting times. To reach the study's objectives, sanitary, economic and social investigations were conducted with the farmers and health workers of the Commune of Adjohoun.

Inquiry was also based on agronomic trial regarding maize yield in the plateau and the plain liable to flooding. The study revealed that soils fertility and farmers income are higher in the plain than that of the plateau. Moreover, the annual prevalence of diseases are globally high in the plain liable to flooding, followed by both plain and plateau and finally by the plateau with the paludism at the top and present at $100 \%$ in the three areas. Then, we have respiratory infections, gastroenteritis and dermatosis with a remarkable presence in the plain and both plain and plateau. Finally, the dermatosis was not recorded in the plateau. Consequently, the annual loss working days due to diseases were globally high in the plain liable to flooding, followed by both plain and plateau.

With regards to this critical situation, the main identified causes are cropping practices and less advisable farmers' behaviors in Todé-gbamè's plain liable to flooding. That is why; it is urging to enhance the technical capacities of the farmers of the study area through a subsequent training.
\end{abstract}


Keywords: cropping practices, yield, diseases, loss working days, Benin

\section{Résumé}

La présente étude se propose de déterminer les prévalences des maladies liées à l'exploitation agricole de la plaine inondable Todé-gbamè au Bénin et à évaluer les pertes de temps de travail y afférentes. Pour atteindre les objectifs de cette étude, des enquêtes socio- économiques et sanitaires ont été effectuées auprès des producteurs et agents de santé de la commune d'Adjohoun. L'enquête a été également appuyée d'un test du rendement du maïs dans le plateau et la plaine inondable. Il ressort de cette étude que la fertilité des terres ainsi que le revenu des producteurs sont plus élevés dans la plaine que dans le plateau. En outre, les prévalences annuelles des maladies sont globalement élevées dans la plaine inondable, suivie de l'ensemble (plaine et plateau) et enfin du plateau avec le paludisme en tête et présent à $100 \%$ dans les trois milieux. Viennent ensuite les infections respiratoires, les gastro-entérites et la dermatose avec une présence remarquable dans la plaine et l'ensemble (plaine et plateau). Enfin, la dermatose n'a pas été enregistrée dans le plateau. En conséquence, les pertes annuelles de jours de travail dues aux maladies sont globalement élevées dans la plaine inondable, suivie de l'ensemble (plaine et plateau). Face à cette situation critique, les principales causes identifiées sont les pratiques culturales et les comportements peu recommandables des producteurs de la plaine inondable de Todé-gbamè. C'est pourquoi, il urge de faire bénéficier aux producteurs du milieu d'étude, un encadrement technique et une formation subséquents.

Mots clés : Pratiques culturales, rendement, maladies, perte de jours de travail, Bénin

\section{Introduction}

L'agriculture, principale activité rurale dans les pays en voie de développement du fait du nombre de personnes qu'elle occupe et du volume de sa production, est l'une des alternatives permettant aux hommes d'assurer leur bien-être. Mais pour faire face à la croissance démographique, les agriculteurs africains doivent relever un défi colossal d'ici l'an 2050 : celui d'augmenter leur production de 300\% (FAO, 1996). En effet, à l'aube du $\mathrm{XXI}^{\mathrm{ème}}$ siècle, une forte proportion de familles africaines vit encore dans l'incertitude alimentaire (IRD, 2010). Selon la FAO (1999), 790 millions d'individus dans le monde en développement sont exposés à ne pouvoir satisfaire leurs besoins alimentaires minima : les deux tiers (2/3) vivent en Afrique, presque un quart en Afrique subsaharienne. D'après Kenmogne et al., (2010), l'Afrique de l'Ouest doit accroître la production à un taux 
minimum de $4 \%$ par an, soit plus de deux fois le taux des décennies précédentes afin d'assurer la sécurité alimentaire. Ceci représente un défi majeur pour l'agriculture béninoise qui, pour ce faire, devra opérer de vigoureux changements dans ses systèmes agraires et dans le mode d'exploitation des terres.

Mais corrélativement, les agriculteurs africains en général, et ceux du Bénin en particulier font face à de nouveaux défis dus aux changements climatiques, notamment une pluviométrie de plus en plus imprévisible de nos jours. En effet, l'analyse des données au Bénin met en évidence une diminution drastique des hauteurs pluviométriques à partir des années 70 et une persistance de ce phénomène au cours des périodes actuelles. Cette variabilité climatique engendre une modification des couverts végétaux et des terres cultivables fertiles favorisant l'avancée de la désertification (Akponikpe, 1999).

Face aux incertitudes du climat couplées à l'épuisement continu des sols des plateaux, la sécurité alimentaire est sérieusement menacée. La culture des versants abrupts des cours d'eau ou l'émigration dans les zones humides en général, devient alors une nécessité impérieuse pour les exploitants agricoles. En effet, selon UICN (2000), les zones humides font partie des écosystèmes les plus productifs de la terre; elles sont tantôt décrites comme les «reins du paysage» pour les fonctions qu'elles remplissent dans les cycles hydrologique et chimique et tantôt comme des «supermarchés biologiques» en raison des vastes chaînes alimentaires et de la riche diversité biologique qu'elles entretiennent. Les bas-fonds et les plaines inondables font de plus en plus l'objet d'une exploitation croissante. La qualité chimique des sols est bonne et l'eau disponible. Elles représentent une alternative économique intéressante pour les paysans béninois qui peuvent y développer une production de sécurité ou de rente (sorgho, maïs, riz) en saison des pluies. En saison sèche, ce sont des zones de pâturage, qui offrent aussi d'intéressantes possibilités de maraîchage, d'arboriculture et de cultures de contre-saison. Pour Atidégla (2012), les plaines inondables et les bas-fonds bénéficient des dépôts d'éléments provenant de l'érosion des versants par les pluies et présentent des sols généralement plus profonds, plus riches et plus humides que ceux du plateau. Le Bénin comporte une multitude de dépressions naturelles qui, du fait de leur morphologie, présentent des caractéristiques hydrologiques particulières faisant de ces zones des régions à haute potentialité agricole. La mise en valeur agricole de ces zones constitue l'une des réponses possibles à la crise actuelle des systèmes traditionnels de production (Sossa, 2001).

Cependant, malgré les multiples fonctions de ces zones, l'impact sanitaire de leur exploitation et les risques environnementaux ne sont pas à négliger. Lavigne et al., (1996), signalent que les zones humides sont des 
lieux répulsifs et malsains. En effet, ces zones abritent des vecteurs qui peuvent contribuer considérablement au développement de maladies touchant les communautés locales. Au Bénin, les maladies comme le paludisme, la bilharziose, les gastro-entérites, les dermatoses pour ne citer que celles-là, y sévissent (Agbossou et Orekan, 2000). Ces différentes maladies ont sans aucun doute des impacts sur la vie des exploitants, la mise en valeur de ces milieux et sur l'environnement. Adjohoun, une Commune de la vallée de l'Ouémé n'étant pas en marge de ces problèmes, mérite une attention particulière à travers l'évaluation des impacts sanitaires qui sont liés à l'exploitation agricole d'une de ses plaines inondables qu'est la plaine inondable Todé-Gbamè.

\section{Materiels Et Methodes}

\section{Cadre d'étude}

Le cadre d'étude est la Commune de Adjohoun qui est du ressort administratif du Département de l'Ouémé. Les travaux ont été conduits sur la plaine Todé-gbamè, située au Sud du village Todé dans la plaine inondable du fleuve Ouémé, dans l'arrondissement d'Azowlissè, un des huit que compte la Commune d'Adjohoun. La population de Adjohoun est très jeune, avec plus de $80 \%$ de personnes âgées de moins de 40 ans (INSAE, 2013). Plus de $80 \%$ de la population active est occupée dans le secteur agricole. La grande plaine Todé-gbamè d'une superficie de 106,26 ha, a fait l'objet de plusieurs aménagements sommaires depuis 1948. Le climat est de type subtropical, caractérisé par de faibles variations de température (moyenne annuelle $27,6^{\circ} \mathrm{C}$ ) et une pluviométrie bimodale : i) une grande saison des pluies d'avril à juillet, ii) grande saison sèche de décembre à mars, iii) une petite saison pluvieuse de septembre à novembre, et iv) une petite saison sèche d'août à septembre. La moyenne pluviométrique annuelle est de $1122,19 \mathrm{~mm}$ an.

Les quatre saisons climatiques du milieu permettent à la Commune d'avoir deux cycles de cultures aussi bien sur le plateau que dans la plaine inondable. Il faut noter que le second cycle de culture (culture de contre saison) est pratiqué pendant la grande saison sèche dans la plaine inondable grâce au retrait des eaux de la crue (la décrue). Le relief de la Commune d'Adjohoun est composé d'un plateau de faible altitude et d'une plaine inondable d'axe Nord-Sud qui, dans la topo séquence Est-Ouest, jouxte le plateau. Elle s'étend de part et d'autre du fleuve Ouémé qui l'inonde annuellement entre les mois de juillet et novembre.

Le territoire de la Commune dispose d'un réseau hydrographique dense dont le plus important cours d'eau est le fleuve Ouémé auquel viennent s'ajouter le confluent Sô, les rivières Tovè, Sissè, les lacs Hlan, Hounhoun propices à l'exploitation halieutique. Les énormes masses d'eaux 
véhiculées pendant les saisons pluvieuses sont encore mal maîtrisées par les populations qui subissent les sinistres qu'elles occasionnent lors des crues exceptionnelles. Le réseau hydrographique de la commune recèle d'énormes potentialités pour l'agriculture de décrue et la pêche que pratiquent les populations. Les sols sont dominés par ceux de la plaine, argileux (argile gonflante) appelée localement « terre noire ». Ce sont des sols hydromorphes organiques. On y rencontre également les sols ferralitiques (terres de barre) très pauvres et à faible rendement.

La végétation a subi une forte dégradation sous l'influence des actions anthropiques à travers l'exploitation agricole et les feux de brousse et est remplacée par des palmeraies. On trouve néanmoins par endroits, de la savane herbacée, de la savane arbustive, des prairies et des marécages dont certains sont en voie de comblement du fait du changement climatique et d'ensablement.

\section{Collecte des données}

\section{Choix de la plaine inondable de Todé-Gbamè et échantillonnage}

L'échantillonnage raisonné a été utilisé pour le choix des ménages agricoles avec pour base la nature des données à collecter et des objectifs à atteindre. En vue d'apprécier l'impact de l'exploitation agricole de la plaine sur la production (rendement), le revenu et les risques d'affections, il a été procédé à un échantillonnage au sein de la plaine inondable et des terres du plateau. Au total, 53 producteurs dont un par ménage ont constitué la base de notre sondage avec trois types de ménages. Il s'agit de ceux qui n'exploitent que la plaine inondable, ceux qui exploitent uniquement le plateau et ceux qui exploitent la plaine et le plateau. Les critères d'échantillonnage des producteurs ont non seulement tenu compte de la zone exploitée mais également des types de cultures. En effet, les principales spéculations pratiquées dans la plaine inondable sont le maïs, riz et les cultures maraîchères (piment, gombo, les légumes feuilles) et sur le plateau, ce sont le maïs et le manioc. Ainsi, les 53 enquêtés sont répartis comme suit : 28 producteurs de maïs et 12 producteurs de piment dans la plaine et 13 qui cultivent l'une ou l'autre au moins des spéculations qui sont produites sur le plateau (Tableau 1).

Tableau 1: Typologie des ménages en fonction des écosystèmes exploités

\begin{tabular}{cccc}
\hline Ecosystème exploité & Cultures & $\begin{array}{c}\text { Nombre de } \\
\text { ménages }\end{array}$ & Total \\
\cline { 1 - 3 } Plaine inondable uniquement & Maïs & 27 & \multirow{2}{*}{15} \\
\cline { 2 - 3 } & Piment & 12 & 13 \\
\hline Plateau uniquement & $\begin{array}{c}\text { maïs et/ou manioc et/ou } \\
\text { arachide }\end{array}$ & 13 & 13 \\
\hline
\end{tabular}




\begin{tabular}{cccc}
\hline $\begin{array}{c}\text { Plaine inondable et Plateau } \\
\text { (Mixte) }\end{array}$ & Maïs & 13 & 13 \\
\hline Total & & 53 & 53 \\
\hline
\end{tabular}

\section{Enquêtes socio-économiques et sanitaires}

Des enquêtes socio-économiques et sanitaires ont été conduites dans la Zone d'étude et ont porté sur les ménages exploitant la plaine inondable Todé-Gbamè et les terres du plateau. Les informations recueillies concernent la production, le rendement du maïs, les revenus agricoles notamment par rapport au maïs et les aspects sanitaires ainsi que les mesures éventuelles prises face aux difficultés rencontrées par les exploitants. Ainsi, l'échantillon de 53 producteurs a été soumis à un questionnaire. Des entrevues ont été ensuite organisées avec les techniciens agricoles, agents de santé, des personnes ressources et des organismes de développement. Outre la littérature existante, l'essentiel du matériel repose sur les fiches de statistiques sanitaires, le questionnaire, le guide d'entretien et une balance de portée $5 \mathrm{~kg}$ pour la mesure du rendement du maïs.

Les données sanitaires par rapport à la prévalence des maladies ont été collectées dans les centres de santé les plus fréquentés par les ménages du village et en particulier par les exploitants de la plaine inondable. Il s'agit donc des données épidémiologiques collectées dans les trois centres de santé que sont: Centre de Santé de Azowlissè, Centre de Santé de Gogbo et Hôpital de Zone d'Adjohoun.

Enfin, pour ce qui est des données relatives aux charges financières annuelles liées aux affections, elles ont été collectées auprès de chaque ménage et une moyenne des coûts a été calculée selon l' écosystème exploité (plaine inondable, plateau ou les deux).

\section{Evaluation du rendement du maïs}

Deux saisons (les deux saisons pluvieuses) sont offertes à l'agriculture sur le plateau tandis qu'en dehors de la grande saison, la plaine inondable offre la culture de décrue. L'agriculture est pratiquée selon les méthodes traditionnelles basées sur la dépendance de la jachère et le faible niveau d'utilisation d'engrais. Les principales spéculations produites sur les plateaux sont le maïs (Zéa mays), le manioc (Manuhot esculenta et l'arachide (Arachis hypogea). Dans la plaine de Todé, c'est le maïs, le piment (Capsicum annuum), le niébé (Vigna unguiculata), le gombo, les légumes feuille et très peu du riz qui sont plus cultivés. Il ressort de ce constat que c'est seulement le maïs qui est produit à la fois sur le plateau et la plaine inondable et c'est pourquoi, pour évaluer la production, nous avons fait le choix de cette spéculation. Ainsi, il s'agira d'analyser les rendements afin de comparer la fertilité et les potentialités de la plaine à celle des terres du 
plateau. Trois paysans ont été suivis de part et d'autre au cours des campagnes agricoles de 2012-2013 pour la grande saison de pluie de Mai à Juillet sur le plateau et la décrue de Décembre à Mars pour la culture de la même variété de maïs DMR de cycle de 90 à 95 jours. Il s'agit des paysans $\mathrm{A}, \mathrm{B}$ et $\mathrm{C}$ pour le plateau et $\mathrm{X}, \mathrm{Y}$ et $\mathrm{Z}$ pour la plaine inondable. La parcelle d'essai retenue par paysan était de $400 \mathrm{~m}^{2}$. Les divers entretiens (sarclage et protection phytosanitaire) ont été identiques dans les deux milieux sauf la fertilisation minérale effectuée au profit du maïs dans le plateau pour la dose paysanne de trois (3) sacs d'engrais NPK de $50 \mathrm{~kg}$ en moyenne par hectare. $\mathrm{Au}$ moment de la récolte, il est procédé à la pesée des produits obtenus au niveau du carré de rendement installé sur chacune des six parcelles. La balance de portée $5 \mathrm{~kg}$ a été utilisée pour peser les grains de maïs obtenus après récolte et égrenage. En effet, après le premier sarclage de chaque parcelle, des carrés de densité y sont posés. Cette opération consiste à délimiter sur chaque parcelle, $1 \mathrm{~m}^{2}$ de parcelle où la densité des plants est la plus forte possible. A la fin du cycle, ces carrés de densité deviennent les carrés de rendement au moment de la récolte et les produits issus de ces carrés sont systématiquement pesés.

\section{Traitement des données}

Les données recueillies ont été dépouillées d'abord manuellement puis traitées à l'ordinateur. Les statistiques sanitaires relevées ont été regroupées suivant la classification internationale des maladies. Les résultats obtenus ont été traduits sous forme de tableaux, des paramètres statistiques (moyenne, écart type, coefficient de corrélation) et des graphiques réalisés au moyen du logiciel Excel. En raison de la non disponibilité de données hydrologiques sur la plaine inondable de Todé-Gbamè, la corrélation entre les prévalences des affections et les précipitations est appréciée à partir des données pluviométriques de la station météorologique d'Adjohoun.

\section{Resultats}

\section{Rendement du maïs produit dans la plaine inondable Todé-Gbamè et dans le plateau}

L'agriculture constitue la principale activité occupant plus de 95\% des ménages interrogés et les principales spéculations pratiquées dans la plaine inondable sont le riz, le maïs et les cultures maraîchères (piment, gombo, les légumes) et sur les plateaux, ce sont le maïs, et le manioc.

Le rendement moyen qui se dégage des essais du maïs au niveau des deux milieux est illustré par la figure 1. 


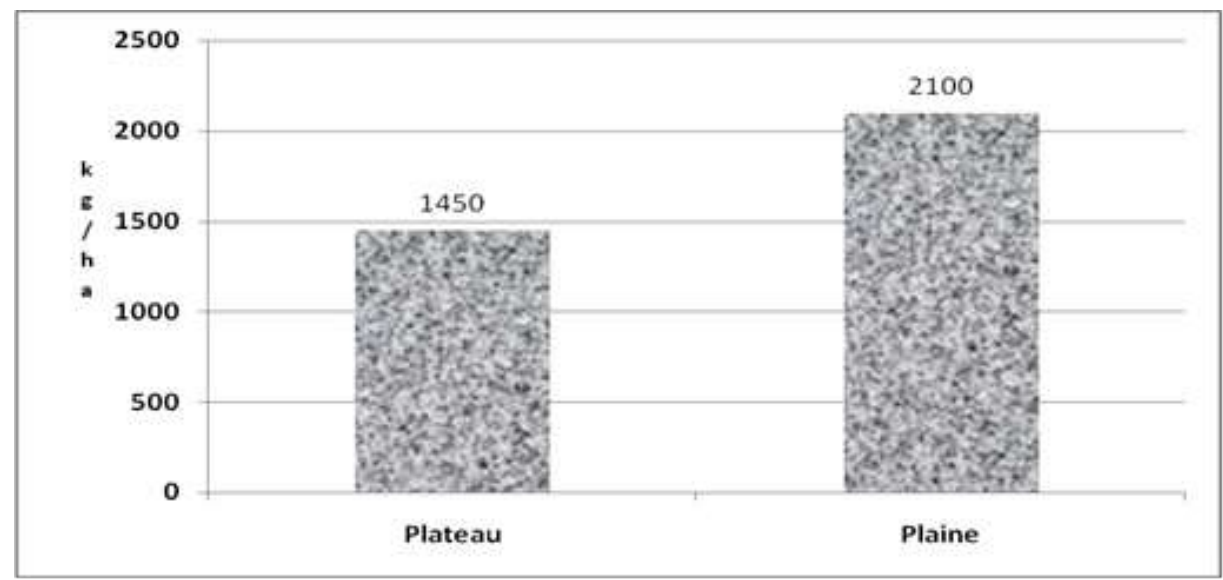

Figure 1 : Rendement moyen du maïs dans la plaine inondable et le plateau

De l'analyse de la figure 1, il ressort que le rendement moyen obtenu sur la plaine est supérieur et presque égal au double de celui obtenu sur le plateau bien que ce ne soit que les producteurs du plateau qui aient fertilisé les sols avec les engrais minéraux $150 \mathrm{~kg}$ de NPK conformément à leur dose traditionnelle. Ceux de la plaine inondable n'ont utilisé aucun fertilisant.

\section{Principales affections enregistrées dans les Centres de Santé retenus}

L'enquête d'évaluation faite auprès des exploitants de la plaine a permis de regrouper la pathologie en sept (7) catégories de classes: i) infections respiratoires aiguës, ii) affections dermatologiques, iii) gastroentérites, iv) affections oculaires, v) affections traumatiques, vi paludisme et vii) autres affections.

Le paludisme, les infections respiratoires aiguës, les gastro-entérites et les affections dermatologiques sont les principales affections signalées dans les Centres de Santé d'Azowlissè, et de Gogbo et à l'Hôpital de Zone d'Adjohoun.

Il ressort de nos investigations que le paludisme est la première cause de consultation avec un taux annuel très élevé de 90\%. Les infections respiratoires aiguës et les gastro-entérites viennent ensuite avec des taux annuels différents selon les centres. Enfin, viennent les dermatoses qui font très peu l'objet des consultations dans les Centres. Les graphes ci-dessous décrivent respectivement l'évolution mensuelle des affections pour l'année 2013 dans les trois centres visités. 


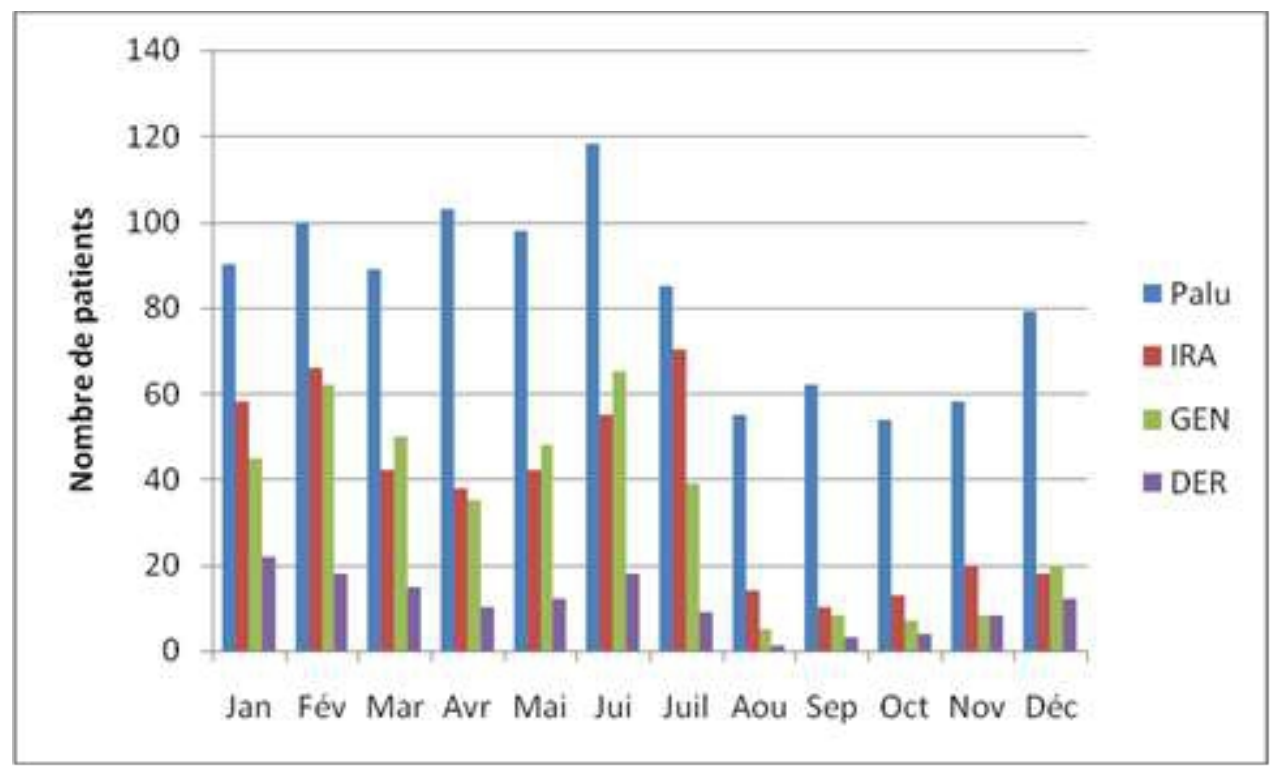

Figure 2 : Evolution mensuelle des affections dans le Centre de santé de Azowlissé Légende $:$ Palu = paludisme, IRA = infections respiratoires aigues, GEN = gastro-entérites, DER $=$ dermatose

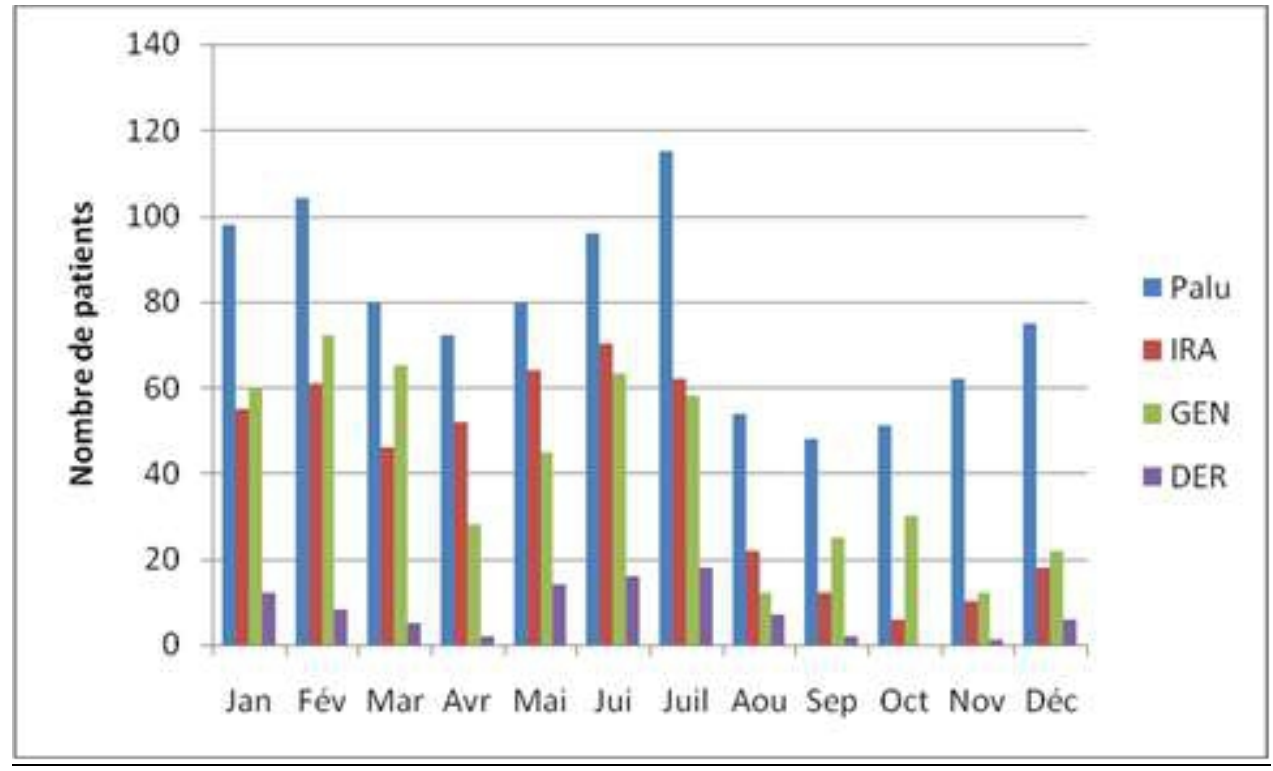

Figure 3 : Evolution mensuelle des affections dans le Centre de santé d'Adjohoun Légende $:$ Palu = paludisme, IRA = infections respiratoires aigues, GEN = gastro-entérites, DER $=$ dermatose 


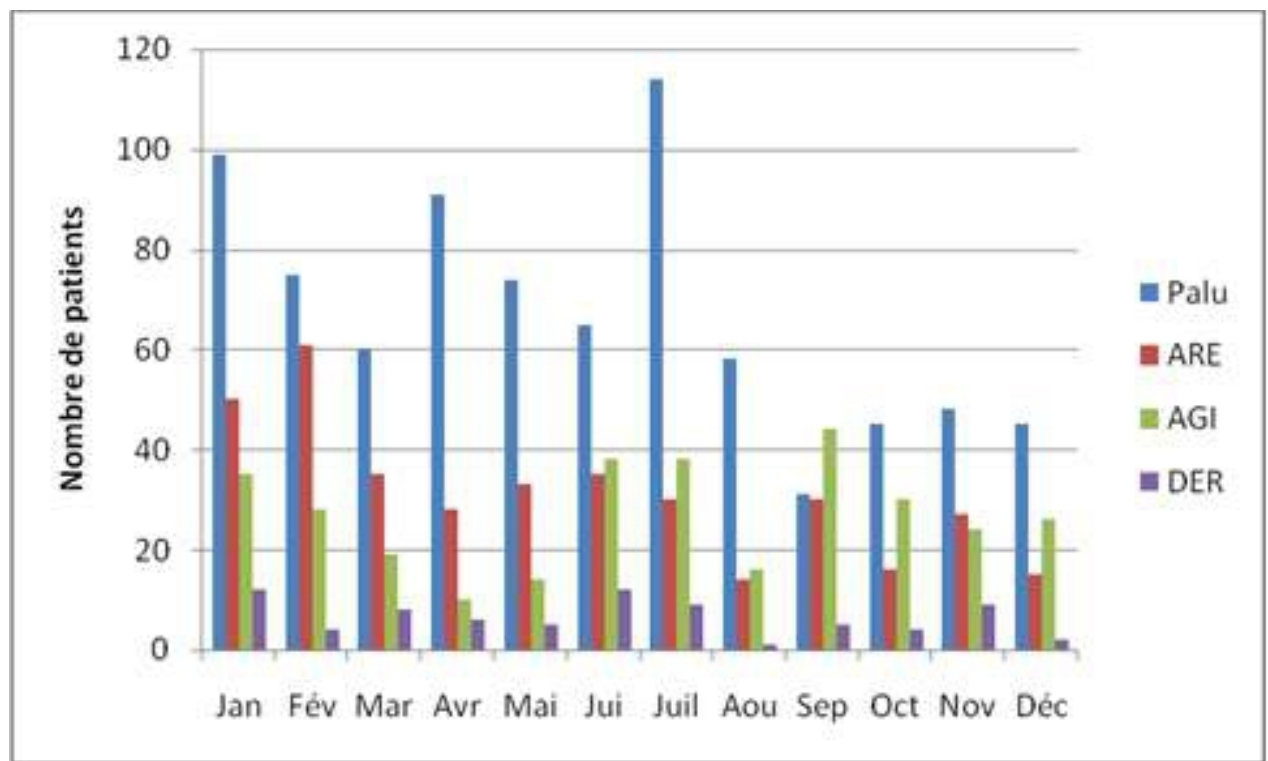

Figure 4 : Evolution mensuelle des affections dans le Centre de santé de Gogbo Légende $:$ Palu $=$ paludisme, $\mathrm{IRA}=$ infections respiratoires aigues, $\mathrm{GEN}=$ gastro-entérites, $\mathrm{DER}=$ dermatose

Des figures 2, 3 et 4 , nous pouvons retenir que le paludisme est la principale affection qui sévit dans le secteur d'étude et que les périodes de fortes occurrences sont les sept premiers mois de l'année (janvier à juillet) pour les 2 Centres de Santé et l'Hôpital de Zone. Ces périodes coïncident avec la grande saison pluvieuse qui est aussi une période d'intenses activités dans la plaine et le plateau. L'effectif le plus élevé des malades est observé dans les mois d'avril et juin avec respectivement 103 et 118 cas recensés à Azowlissè ; dans les mois de février et juillet avec respectivement 104 et 115 cas enregistrés à Adjohoun et enfin dans les mois de janvier et juillet à Gogbo avec 99 et 114 cas recensés. Les infections respiratoires sont constatées en période de décrue. L'effectif le plus élevé est observé dans les mois de juillet, février, janvier et juillet avec respectivement 70, 64, 58 et 55 cas à Azowlissè ; dans les mois de juin, mai, juillet et février avec respectivement 70,64, 62 et 61 cas recensés à Adjohoun et enfin dans les mois de février et janvier avec respectivement 61 et 50 cas recensés à Gogbo. Pour ce qui est des gastro-entérites, les périodes de fortes occurrences sont les mois de janvier à juillet à Azowlissè ; de janvier à mars et d'avril à juillet à Adjohoun et les mois de janvier, juin, juillet et septembre à Gogbo. Les moyennes annuelles des gastro-entérites dans ces trois Centres étant respectivement 33, 42 et 27 . Enfin, les dermatoses qui font très peu l'objet des consultations, sont fréquentes toute l'année dans les deux premiers Centres sans périodes d'occurrences particulières avec des cas mensuels 
faibles. Elles sont presque absentes dans le centre de Gogbo avec un effectif total de 77 pour le compte de l'année.

En résumé, de toutes les affections qui prévalent dans la zone d'étude, ce sont le paludisme, les infections respiratoires et les gastroentérites qui font plus l'objet de fréquentes consultations. Ensuite, viennent les dermatoses qui sont peu évoquées.

\section{Affections liées aux milieux exploités (plaine inondable, plateau ou les deux)}

La maladie la plus évoquée par les ménages enquêtés est le paludisme. Toutefois, les infections respiratoires aiguës et les gastro-entérites ont été aussi recensées. Pour évaluer l'impact de la mise en valeur des terres sur l'état de santé des exploitants, la répartition des cas de maladies dans les ménages a été faite en tenant compte du milieu exploité.

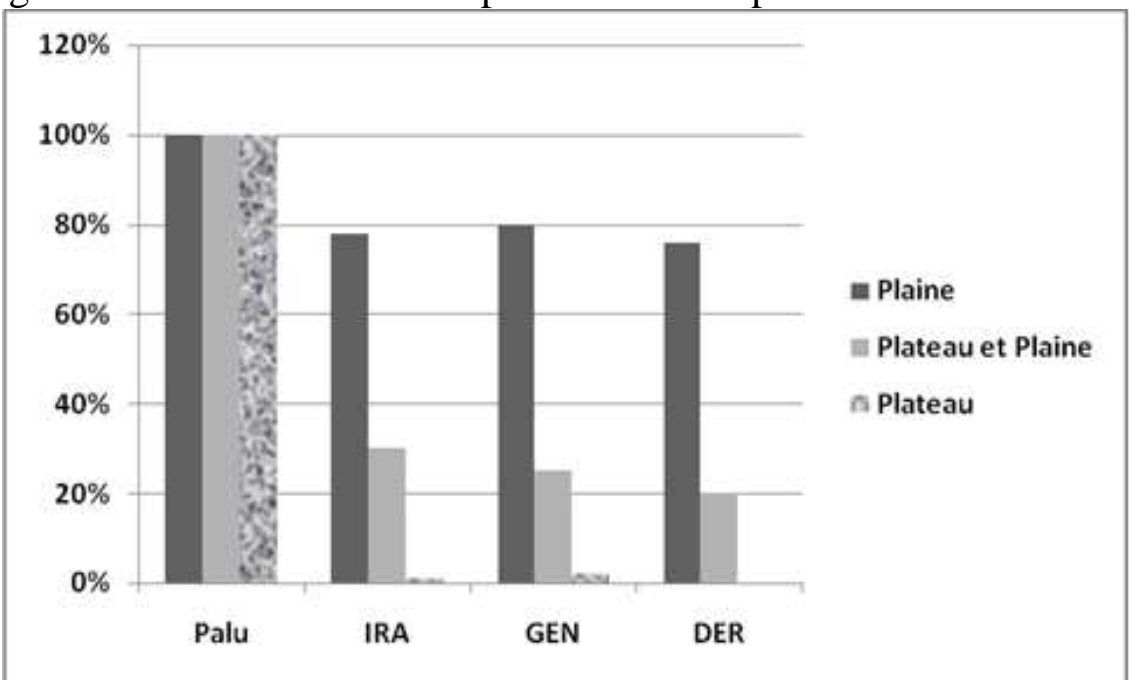

Figure 5: Répartition des principales maladies en fonction du milieu exploité Légende $:$ Palu $=$ paludisme, IRA $=$ infections respiratoires aigues, $\mathrm{GEN}=$ gastro-entérites, DER $=$ dermatose

De la figure 5, il ressort que les exploitants agricoles de la plaine et ceux du plateau n'ont en commun que le paludisme. Les infections respiratoires aiguës et les gastro-entérites ainsi que les dermatoses sont absentes chez les producteurs qui cultivent uniquement dans le plateau. Par contre, ces affections menacent ceux qui sont à la fois sur les deux milieux, mais dans de faibles proportions. Le test de Chi-Square appliqué à ce résultat afin de voir s'il y a dépendance entre ces affections et le milieu a donné les résultats suivants :

Chi-Sq $=19,594 ; \mathrm{DF}=3 ; \mathrm{P}-$ Value $=0,000$ 
La probabilité associée à ce test $(0,000)$ étant inferieure à 0,05 , nous rejetons l'hypothèse nulle et nous concluons qu'il existe statistiquement une dépendance très hautement significative entre les affections et la plaine inondable.

\section{Revenus des ménages par rapport à la production du maïs}

Une activité ne mérite d'être encouragée, que si elle permet un gain supplémentaire de revenus. Il s'agit ici de savoir si la mise en valeur de la plaine inondable induit vraiment ce gain supplémentaire pour les ménages. Néanmoins, nous nous proposons de voir à travers cette étude si actuellement, la mise en valeur de la plaine améliore le revenu des exploitants qui produisent le maïs.

Tableau 2: Marge brute moyenne des exploitants échantillonnés par culture selon les zones.

$$
\text { Culture Marge brute moyenne (FCFA/ha) }
$$

\begin{tabular}{cc}
\hline Maïs / plaine inondable & 460000 \\
\hline Maïs / plateau & 250000 \\
\hline
\end{tabular}

L'analyse du tableau révèle qu'il y a une différence entre les revenus avec la plaine inondable qui a généré plus de revenus que le plateau.

Tableau 3 : Test statistique de comparaison des moyennes

\begin{tabular}{cc}
\hline T-Test of difference & 0 (vs not) \\
\hline T-Value & 7,25 \\
\hline P-Value & 0,000 \\
\hline DF & 13 \\
\hline
\end{tabular}

D'après le tableau 3, l'analyse statistique des résultats montre que les différences de revenus sont très hautement significatives au seuil de $1 \%$. Cela est sans doute dû à la bonne fertilité des terres de la plaine et à l'alimentation régulière en eau de ces dernières, contrairement aux terres du plateau.

De même, sur la plaine de Todé, la première culture qui rapporte d'importants revenus pour les producteurs est le piment. En effet, les enquêtes ont révélé que par campagne, la marge brute moyenne par hectare pour le piment est de deux millions cinq cent mille (2 500 000) francs CFA. Ceci justifie l'intérêt des producteurs à s'adonner tous à la production du piment dans la plaine. A ces cultures s'ajoutent les légumes feuilles, suivi du gombo.

Les revenus tirés de l'exploitation de la plaine inondable, sont utilisés à diverses fins : plus de la moitié $(56 \%)$ assurent la scolarisation des enfants et la santé de toute la famille, une partie (28\%) est réservée pour agrandir le champ de production et améliorer les outils de production, une partie (11\%) pour les cérémonies (enterrement) et enfin la dernière partie (4\%) pour la dot de la nouvelle femme et l'achat d'autres biens. Les producteurs investissent 
moins leur revenu dans l'alimentation de la famille car plus de $80 \%$ des vivres entrant dans leur alimentation sont produits par eux-mêmes. Aussi, ces derniers n'ont-ils pas de problèmes de logement car ils logent tous dans des maisons familiales ou construites par eux-mêmes. Rappelons aussi que près de $55 \%$ de ces producteurs sont des pêcheurs dans la plaine en période de crue, et cette activité leur rapporte en moyenne (250 000 F CFA) par an. De plus, ces producteurs ne connaissent pas de période morte au cours de l'année. Selon la saison et le milieu exploité, ils sont soit dans l'agriculture, soit dans la pêche, soit dans la transformation du vin de palme en Sodabi (boisson alcoolisée) ou de la noix de palme en huile végétale ou soit dans leur métier de base (13\% : menuiserie, maçonnerie ou autres).

En somme, l'exploitation de la plaine inondable même «non aménagée» permet aux producteurs de satisfaire au mieux les besoins fondamentaux de la vie.

\section{Perte de jours de travail pour cause de maladie}

La maladie a d'autres coûts indirects que l'exploitant doit gérer. Ainsi, ce dernier est presque toujours contraint de suspendre ses activités pendant une période donnée ; ce qui est préjudiciable pour son bien être. L'incapacité de travailler pour cause de maladie liée à l'environnement est observée sur la plaine de Todé. Les pertes en jours d'activités, sont principalement dues au paludisme et quelques fois aux infections respiratoires et gastro-entérites (Tableau 4).

Tableau 4 : Importance de perte de jours de travail

\begin{tabular}{cccc}
\hline $\begin{array}{c}\text { Nombre de jours perdus } \\
\text { par maladie }\end{array}$ & Paludisme & $\begin{array}{c}\text { Infections } \\
\text { respiratoires }\end{array}$ & $\begin{array}{c}\text { Gastro- } \\
\text { entérites }\end{array}$ \\
\hline $\begin{array}{c}\text { Exploitants de la plaine } \\
\text { inondable }\end{array}$ & 12 & 9 & 4 \\
\hline Exploitants du plateau & 9 & 0 & 0 \\
\hline
\end{tabular}

Du tableau 4, il ressort que les exploitants de la plaine perdent en moyenne 12 jours pour cause de paludisme contre 9 jours pour ceux du plateau. Les maxima de jours perdus recensés lors de nos enquêtes sont de 18 et 10 jours respectivement pour les exploitants de la plaine et du plateau de décembre 2012 à décembre 2013.

En ce qui concerne les infections respiratoires et les gastro-entérites, elles ne posent pas de problèmes aux producteurs du plateau puisqu'ils n'en souffrent

pas. Mais les exploitants de la plaine perdent en moyenne 9 jours pour les infections respiratoires et 4 pour les gastro-entérites avec des maxima de 20 et 14 jours respectivement. En effet, les exploitants de la plaine seraient en activité presque toute l'année (9 mois d'agriculture et presque 3 mois de pêche), si toutes ces affections n'avaient pas des répercussions sur leur santé. 
Or, les enquêtes ont révélé que ces maladies, en particulier les infections respiratoires et les gastro-entérites, surviennent au cours des travaux agricoles et engendrent des dépenses supplémentaires (de main-d'œuvre) aux producteurs et une perte de temps.

\section{Charges financières annuelles liées aux affections}

Pour la plupart des affections, les malades sont d'abord traités à l'indigénat ou à l'automédication avant que les chefs de ménage ne pensent aux soins modernes. Ces moyens de traitement présentent certains risques pour la santé des exploitants. Mais bien qu'étant conscients de cela, la plupart des ménages évoquent le problème de ressources financières qui les contraint à essayer d'abord la thérapie traditionnelle.

L'enquête qualitative réalisée lors de nos travaux de recherche révèle que les sources de revenus permettant aux ménages de recevoir les soins médicaux sont essentiellement agricoles. Bien que l'accès aux soins modernes paraisse un peu contraignant, les exploitants de la plaine inondable ne s'en plaignent pas trop. Ils disposent d'un revenu assez élevé, tiré des activités pour bénéficier des soins médicaux primaires c'est-à-dire ceux de base (accouchement et consultation pour les maladies courantes).

Nos enquêtes ont montré que les exploitants de la plaine dépensent en moyenne $90000 \mathrm{~F} \mathrm{CFA} /$ an pour la santé de la famille. En général, le constat fait est qu'aucune somme fixe n'était destinée spécifiquement aux soins de santé, la plupart des ménages ont expliqué que les revenus issus des ventes de produits agricoles, sont généralement utilisés pour tous les besoins et qu'ils s'en servent pour gérer les problèmes de santé comme cela pourrait être le cas de n'importe quels autres imprévus. Au niveau des exploitants mixtes, les charges financières de santé tournent autour de 50000 F CFA l'année alors que dans le plateau, elles avoisinent annuellement les 25000 F CFA.

\section{Discussion}

Impacts positifs de l'exploitation agricole de la plaine inondable TodéGbamè sur le rendement du maïs

La dose d'engrais chimique apportée par les producteurs est inférieure à celle recommandée par l'Institut National des Recherches Agricoles du Bénin (INRAB). Malgré l'apport de cette dose paysanne d'engrais minéraux (trois sacs d'engrais NPK) au maïs du plateau, le rendement était presque la moitié de celui obtenu dans la plaine inondable. Ceci est la preuve de la bonne fertilité des terres de la plaine inondable. En effet, selon Akondé et al., (2005) de l'INRAB, il faut pour la production d'un hectare de maïs, $200 \mathrm{~kg}$ de NPK et $100 \mathrm{~kg}$ d'urée, pour s'attendre à obtenir au moins 2t/ha lorsque tout l'itinéraire technique est bien suivi. Le 
non respect de la dose d'engrais par les producteurs serait lié au manque de ressources financières. Somme tout, il découle de ces essais que les terres de la plaine sont plus productives que celles du plateau car leur fertilité est renouvelée annuellement et de façon naturelle grâce aux matières organiques, aux alluvions apportés par la crue. Les mêmes résultats ont été obtenus par Agbossou et al., (2005) dans les bas-fonds de Logbogba (Bénin), où les enquêtes ont montré que les terres de bas-fonds sont plus riches que celles des plateaux. Les résultats obtenus par Agbodjogbe (2008) dans le basfond Aïzo, commune de Ouinhi, révèlent les mêmes tendances pour le riz et les autres cultures vivrières. Toutefois, les causes probables sont les spécificités de ces milieux dont notamment la fertilité de leurs sols. Mais signalons aussi que de plus en plus les producteurs des plaines et bas-fonds sont souvent confrontés aux problèmes d'inondation qui leur créent parfois d'énormes pertes et influent négativement sur la production en régime pluvial. Enfin, la plaine inondable permet une grande diversification des produits agricoles. En effet, d'autres spéculations plus rentables en dehors de celles communes aux deux milieux y sont produites. Il s'agit des produits maraîchers (piment, gombo, légumes feuilles etc.) et du riz.

\section{Responsabilité des producteurs dans la prévalence des maladies enregistrées}

Conformément aux résultats d'enquête ainsi qu'aux analyses statistiques, nous pouvons affirmer que les maladies enregistrées dans la zone d'étude notamment dans la plaine sont pour la plupart liées aux activités dans la plaine inondable. Ces résultats sont en phase avec ceux de Vitchoékè (2006) dans la commune de Covè et de Bonou (2005) dans les Arrondissements de Kpanrou et de Zinvié dans la Commune d'AbomeyCalavi. Certes certaines maladies comme le paludisme, les gastro-entérites et les dermatoses sont propres à la plaine mais d'autres comme les infections respiratoires pourraient en avoir d'autres. C'est pourquoi, d'après Adéchian et al., (2015), la présence des infections respiratoires dans la plaine peut s'expliquer par l'utilisation sans précaution d'insecticides et de pesticides dans la lutte contre les ravageurs. En effet, la pulvérisation des produits pesticides (insecticides et herbicides) se fait dans des conditions peu recommandables c'est-à-dire sans aucune protection (gants, cache-nez, bottes, etc.). De plus, les infections respiratoires sont beaucoup plus présentes selon les données recueillies dans les centres de santé, pendant la décrue où les activités agricoles sont fortes (Ahouangninou et al., 2011). Quant à la présence des gastro-entérites dans la plaine inondable, cela peut être également dû au manque d'hygiène dans les ménages (Kenmogne et al., 2010). En effet, les besoins de se mettre à l'aise (défécation) se font à l'air libre dans la plaine. Malgré la source d'eau potable dont bénéficie le village, 
l'eau de la plaine constitue encore une eau de boisson pour les producteurs. Le manque d'hygiène par rapport aux aliments peut entrainer également les cas de diarrhées observés sur la plaine. Par exemple, les repas sont souvent non couverts, laissés aux soins de la poussière, des mouches et de tous les microbes circulants dans l'atmosphère. Il en est de même de l'eau de la plaine servant d'eau de boisson après le repas. Enfin, la présence des dermatoses dans le milieu est due au contact direct avec l'eau souillée de la plaine.

\section{Impacts négatifs des maladies sur le temps de travail et le revenu des ménages}

La maladie a d'autres coûts indirects que l'exploitant doit gérer. Ainsi, ce dernier est presque toujours contraint de suspendre ses activités pendant une période donnée ; ce qui est préjudiciable pour son bien être. L'incapacité de travailler pour cause de maladie liée à l'environnement est observée sur la plaine de Todé.

En effet, les chiffres enregistrés sont supérieurs à ceux trouvés (7 et 5) par Agbodjogbe (2008) sur le bas-fond et le plateau Aïzo. Dans la zone d'étude, pour remédier à ce facteur limitant qu'est la maladie, ces producteurs vont prendre des injections ou des sérums (pour ceux qui ont les moyens) dans les Centres de Santé avant chaque campagne. D'autres sont soumis aux régimes traditionnels avec des tisanes des plantes médicinales avant de faire recours à l'hôpital en cas de non-satisfaction.

Les traitements similaires sont observés pour le paludisme qui est l'affection la plus répandue dans toute la zone d'étude et qui est beaucoup crainte parce que accompagnée parfois d'une fièvre mortelle. Celle-ci se retrouve en particulier chez les enfants de moins de 6 ans (Butz, 1984). En effet, la majorité des producteurs (plus de 50\%) enregistrent assez de cas de décès de leurs enfants atteints de cette fièvre comme signalé par Daigl (2002). C'est pourquoi les producteurs font beaucoup d'enfants afin de s'offrir plus de chance d'en disposer. Par ailleurs, signalons que les démangeaisons épidermiques (passagères) dont se plaignent les exploitants de la plaine influencent peu les travaux agricoles.

\section{Conclusion}

La présente étude vient de montrer que la plaine inondable constitue une importante source de potentialités agricoles pour la population mais également un milieu à risques sanitaires élevés. En effet, les enquêtes ont révélé que les maladies comme le paludisme, les infections respiratoires, les gastro-entérites et les dermatoses menacent les producteurs et constituent un frein pour les activités lorsqu'elles surviennent. Toutefois, en dehors des problèmes d'inondation qui causent d'énormes dégâts sur les cultures, il a été 
déploré au niveau de certaines pratiques agricoles observées sur la plaine quelques risques pour la santé des producteurs et consommateurs des produits maraîchers ainsi que pour l'environnement. Il s'agit entre autres de l'utilisation inappropriée et sans précautions des produits phytosanitaires en l'occurrence les pesticides sur les cultures pour lutter contre les ravageurs. Enfin, eu égard à ce qui précède, une gestion durable de la plaine est nécessaire. Pour ce faire, nous formulons à l'intention des producteurs et décideurs les suggestions suivantes : i) assurer un encadrement technique des producteurs ; ii) faciliter leur accès aux crédits ; iii) doter les producteurs en équipements de protection; iv) promouvoir l'utilisation des biopesticides/bio-insecticides ; v) assurer une formation et un suivi régulier en matière d'hygiène pour les producteurs et vi) réaliser un aménagement total de la plaine afin de ne pas déplacer les problèmes corrigés.

\section{References:}

1. Adechian, S. A., Baco, N. M., Akponikpe, I., Toko I. I., Egah, J., et Affoukou, K. (2015). Les pratiques paysannes de gestion des pesticides sur le maïs et le coton dans le bassin cotonnier du Bénin, VertigO - la revue électronique en sciences de l'environnement, Vol. 15, $\mathrm{N}^{\circ}$ 2, [En ligne], URL : http://vertigo.revues.org/14976 ; DOI : 10.4000/vertigo. 14976

2. Agbodjogbe, J. G. (2008). Impacts socio-économiques et environnementaux de la mise en valeur du bas-fond Aïzè commune de OUINHI, département du ZOU. Thèse d'Ingénieur Agronome, UAC/FSA/AGRN. 106 p.

3. Agbossou, E.K., et Orekan V.O.A. (2000). Impacts sanitaires des aménagements de bas-fonds sur les populations : cas des bas-fonds de Gomé et de Tré dans le département du Zou au Bénin. Colloque international «Eau et Santé »-Ouaga.

4. Agbossou, E., Ahamide, B., Hounsou, M., et Doussouhoin, F. (2005). Rapport d'étude socio-économique de l'aménagement de 100 ha de bas-fond dans la commune de Covè. 22 p.

5. Ahouangninou, C., Fayomi, B.E., et Martin T. (2011). Évaluation des risques sanitaires et environnementaux des pratiques phytosanitaires des producteurs maraîchers dans la commune rurale de Tori-Bossito (Sud-Bénin), Cahiers Agricultures, 20, pp. 216-222

6. Akondé, T. P., Toukourou A. M., et Aihou K. (2005). La culture du maïs est possible dans un champ infesté de striga, Référentiel Technico-économique de la production agricole, $56 \mathrm{p}$.

7. Akponikpe, P. B. I. (1999). Contribution à l'évaluation de l'impact des changements climatiques sur la production agricole : cas du maïs 
(Zea mays L.) au Sud Bénin. Thèse d'Ingénieur Agronome. FSA/UNB, Bénin, 89 p.

8. Atidegla, C. S. (2012). Rapport d'étude des techniques d'aménagement des bas-fonds d'Allada et d'Abomey-Calavi au Bénin. Centre Régional pour la Promotion Agricole (CeRPA) Atlantique-Littoral, $35 \mathrm{p}$.

9. Bonou, M. (2005). Impact Socio sanitaire de l'Exploitation Agricole des terres Humides au Sud Bénin dans les arrondissements de Kpanroun et Zinvié (Commune d'Abomey-Calavi). Mémoire de Maîtrise, FLASH/UAC, 97 p.

10. Butz, W. P., Habicht J. P., et Davanzo (1984). Environmental Factors in the Relationship between Breastfeeding and Infant Mortality: The Role of Sanitation and Water in Malaysia, American Journal of Epidemiology, Vol. 119, No. 4, pp. 516-525.

11. Daigl M. (2002). Evaluation des Facteurs de Risque Pour le Paludisme et la Diarrhée dans les Quartiers Yaoséhi, Niangon Sud Sicogi et le village d'Azito, Commune de Yopougon, Abidjan, Côte d'Ivoire. Mémoire en Sciences, Schweiz," Tropeninstitut, University Basel, 2002, $108 \mathrm{p}$.

12. FAO, (1996). Rapport du sommet mondial de l'alimentation. Novembre 96, Rome, Italie, 152 p.

13. FAO, (1999). Rapport sur l'état de l'insécurité alimentaire dans le monde. L'insécurité alimentaire : la faim au quotidien et la crainte permanente de la famine. $\mathrm{p} 35$

14. Kenmogne G. R. K., Rosillon F., Mpakam H. G. et Nono A. (2010). Enjeux sanitaires, socio-économiques et environnementaux liés à la réutilisation des eaux usées dans le maraîchage urbain : cas du bassin versant de l'Abiergué (Yaoundé-Cameroun). VertigO - la revue électronique en sciences de l'environnement, Vol. $10 \mathrm{~N}^{\circ} 2$, [En ligne], URL : http://vertigo.revues.org/14976 ; DOI : 10.4000/vertigo. 14976

15. INSAE (Institut National de la Statistique et de l'Analyse Economique), (2013). Troisième Recensement Général de la population et de l'Habitation: Statistiques démographiques. Bénin, Tome 3, $35 \mathrm{p}$,

16. IRD (Institut de Recherche pour le Développement), (2010). Impacts sanitaire et nutritionnel des hydro-aménagements en Afrique". Colloque international Eau et Santé. Ouagadougou, Burkina Faso,

17. Lavigne D., Boucher L., et Vidal L. (1996). Les bas-fonds en Afrique tropicale humide : stratégies paysannes, contraintes agronomiques et 
aménagements. Fertilité du milieu et stratégies paysannes sous les tropiques humides. Actes du séminaire international. CIRAD, $161 \mathrm{p}$

18. Sossa, B. (2001). Contribution à la mise en valeur de la plaine d'inondation du lac Toho à Kponou ( $\mathrm{S} / \mathrm{P}$ Athiémé, Département du Mono), Thèse d'Ingénieur Agronome, FSA/UNB, $84 \mathrm{p}$.

19. UICN, (2000). Union internationale pour la conservation de la nature et de ses ressources Groupe d'experts des plaines d'inondation sahéliennes. Vers une gestion durable des plaines d'inondation sahéliennes. UICN, Gland, Suisse et Cambridge, Royaume-Uni. xii + $214 p$.

20. Vitchoeke J. M. (2006). Evaluation Socio économique et Sanitaire de la mise en Valeur du Bas-fond Ouantégo de la commune de Covè. Thèse d'Ingénieur Agronome, UAC/FSA/AGRN, 104p. 